\title{
Anisotropic magnetoconductivity of a three-dimensional disordered electron gas
}

\author{
Alba Theumann and S. G. Magalhães \\ Instituto de Física, Universidade Federal do Rio Grande do Sul, Caixa Postal 15051, 91500 Porto Alegre, \\ Rio Grande do Sul, Brazil \\ (Received 17 August 1988)
}

\begin{abstract}
The magnetoconductivity of a three-dimensional disordered electron gas is calculated from first principles with use of the exact electron eigenstates in a magnetic field, thus avoiding the usual semiclassical approximation in this field. Our calculation gives anisotropic components for the conductivity tensor, and the isotropic behavior predicted by other authors is recovered in the limit $\omega_{c} \tau \ll<$, where $\omega_{c}$ is the cyclotron frequency and $\tau$ the elastic lifetime.
\end{abstract}

\section{INTRODUCTION}

The results for the magnetoconductivity of a very disordered electron gas in two ${ }^{1}$ and three ${ }^{2}$ dimensions were reported shortly after Abrahams et al. ${ }^{3}$ formulated the scaling theory of localization. The detailed calculations only appeared in more recent review articles, ${ }^{4-6}$ giving as a result a perfectly isotropic conductivity tensor, while by general symmetry arguments we would expect that in the presence of a magnetic field $B$ in the $z$ direction the diagonal components of the tensor satisfy the inequality $\sigma_{x x}=\sigma_{y y} \neq \sigma_{z z}$. The approximations made in these papers can be summarized as follows: ${ }^{5,6}$ first, they calculated the isotropic conductivity in the absence of an external field; second, the magnetic field was switched on phenomenologically by replacing $(1 / 2 m)\left(\mathbf{k}_{\perp}+\mathbf{k}_{\perp}^{\prime}\right)^{2}$ $\rightarrow \omega_{c}^{*}\left(n+\frac{1}{2}\right)$ in the diffusion propagator. Here $\omega_{c}^{*}=e^{*} B / m$ is the cyclotron frequency for one particle of charge $e^{*}=2 e, n$ is the Landau level index, and $\mathbf{k}_{\perp}$ indicates the projection of the momentum $\mathbf{k}$ on the $x-y$ plane. It followed from this that the calculated conductivity remained isotropic in the presence of the field, although it is not obvious that it will be so in a calculation based from the start on the Landau levels that are the exact electron eigenfunctions.

To investigate this point we present here an alternative calculation of the three-dimensional conductivity based on the Landau eigenstates, thus avoiding the semiclassical approximation for the Green's function that is usually done in this problem. ${ }^{5,6}$ This approach has been followed previously to study interaction effects in the twodimensional magnetoconductivity, ${ }^{7}$ although to our knowledge it has not yet been used to obtain the contribution of the multiply crossed diagrams ${ }^{3}$ in the weakly localized regime.

The sum of these diagrams in the presence of a field is not an obvious extension of the results when $B=0$, due to the presence of noncanceling phases that destroy the translational invariance of the propagators. To show the difficulties inherent in a calculation of the conductivity in a system that lacks translational invariance we go into some detail in Sec. 2 and the Appendix.

We show in Sec. III that the relevant vertex function satisfies an integral equation that we solve to $O\left(\omega_{c}^{2}\right)$ and in the diffusion pole approximation that is usual in this problem. $^{3}$

These are the only approximations involved in our work and our results allow us also to discuss the relevance of the inelastic lifetime ${ }^{4-6} \tau_{i}$ in three dimensions. While the expression for the conductivity in two dimensions has an infrared divergence that forces the phenomenological introduction of a cutoff interpreted as $\tau_{i}^{-1}$, the integrals in three dimensions are perfectly convergent in the infrared and a cutoff is mathematically unnecessary, thus we present in Sec. III the results for $\tau_{i}^{-1}=0$.

This leads to a discussion of time scales, as our results in Sec. III for $\tau_{i}^{-1}=0$ predict an anisotropic behavior of the conductivity tensor for very high fields $\omega_{c}>\tau^{-1}, \tau$ being the elastic lifetime, while the predicted isotropic behavior in $\left(\omega_{c}\right)^{1 / 2}$ prevails for all fields that satisfy $0<\omega_{c} \tau<1<D_{0}^{1 / 3}$. The introduction of a finite $\tau_{i}$ would modify this asymptotic behavior, predicting a dependence in $\omega_{c}^{2}$ for very low fields, $0<\omega_{c}<1 / \tau_{i} D$, while the $\left(\omega_{c}\right)^{1 / 2}$ behavior would be observed for $1 / \tau_{i} D_{0}<\omega_{c}$ $<1 / \tau D_{0}$, where $D_{0} \gg 1$ is the diffusion coefficient.

We present in Sec. II the general expression for the diagonal components of the conductivity tensor and the calculation of the vertex function that sums the multiple crossed diagrams in the presence of the magnetic field, to lowest order in the field. Section III is dedicated to the detailed calculation of the conductivity and Sec. IV to discussions.

\section{GENERAL FORMULATION}

The system under study is a gas of electrons in the presence of a magnetic field $\mathbf{B}=\boldsymbol{\nabla} \times \mathbf{A}_{0}$ in the $z$ direction that are scattered by the random impurity potential $V(\mathbf{r})$ with zero mean, and variance

$$
\left\langle V(\mathbf{r}) V\left(\mathbf{r}^{\prime}\right)\right\rangle=U \delta\left(\mathbf{r}-\mathbf{r}^{\prime}\right) .
$$

The Hamiltonian is

$$
H=-\frac{1}{2} \int d \mathbf{r} \psi^{\dagger}(\mathbf{r}) \mathbf{D}_{r}^{2} \psi(\mathbf{r})+\int d \mathbf{r} \psi^{\dagger}(\mathbf{r}) \boldsymbol{V}(\mathbf{r}) \psi(\mathbf{r}),
$$


where the field operators $\psi(\mathbf{r}), \psi^{\dagger}(\mathbf{r})$ satisfy the usual fermion anticommutation rules. Spin indices can be neglected in the absence of spin orbit and paramagnetic interactions. We work in units $\hbar=m=c=1$, and $e$ is the electron charge. We also have in Eq. (2)

$$
\mathbf{D}_{r}=\nabla_{r}-i e \mathbf{A}_{0}(\mathbf{r}),
$$

and in the Landau ${ }^{8}$ gauge

$$
\mathbf{A}_{0}(\mathbf{r})=(-B y, 0,0) \text {. }
$$

The linear-response formalism ${ }^{9}$ gives, for the components of the conductivity tensor,

$$
\begin{aligned}
\sigma_{\mu \nu}(\mathbf{q}, \omega)=\frac{e^{2}}{4 \pi \omega} \int_{-\omega}^{0} d \varepsilon \int & d\left(\mathbf{r}-\mathbf{r}^{\prime}\right) e^{i \mathbf{q} \cdot\left(\mathbf{r}-\mathbf{r}^{\prime}\right)} \\
& \times \Gamma_{\mu \nu}\left(\mathbf{r}-\mathbf{r}^{\prime}, \varepsilon, \omega\right),
\end{aligned}
$$

where $\mu, v$ indicate the space directions $x, y$ or $z$ and

$$
\begin{aligned}
& \Gamma_{\mu v}\left(\mathbf{r}, \mathbf{r}^{\prime}, \varepsilon, \omega\right) \\
& \quad=\left[\left(D_{\rho}^{\mu}-D_{r}^{\mu *}\right)\left(D_{\rho^{\prime}}^{v}-D_{r^{\prime}}^{v *}\right) \Pi_{\varepsilon, \omega}\left(\mathbf{r}, \mathbf{r}^{\prime} \mid \boldsymbol{\rho}, \boldsymbol{\rho}^{\prime}\right)\right]_{\mathbf{r}=\rho, \mathbf{r}^{\prime}=\boldsymbol{\rho}^{\prime}} .
\end{aligned}
$$

The polarization in Eq. (6) is given by

$$
\Pi_{\varepsilon, \omega}\left(\mathbf{r}, \mathbf{r}^{\prime} \mid \boldsymbol{\rho}, \boldsymbol{\rho}^{\prime}\right)=\left\langle\widetilde{G}_{\varepsilon+\omega}\left(\mathbf{r}, \mathbf{r}^{\prime}\right) \widetilde{G}_{\varepsilon}\left(\boldsymbol{\rho}^{\prime}, \boldsymbol{\rho}\right)\right\rangle,
$$

and the bracket in Eq. (7) indicates the average over random impurities. The one-particle Green's function is the solution of the equation

$$
\left[\omega+\varepsilon_{F}+\frac{1}{2} \mathbf{D}_{r}^{2}-V(\mathbf{r})\right] \widetilde{G}_{\omega}\left(\mathbf{r}, \mathbf{r}^{\prime}\right)=\delta\left(\mathbf{r}-\mathbf{r}^{\prime}\right) .
$$

In the presence of a magnetic field, the polarization $\Pi_{\varepsilon, \omega}\left(\mathbf{r}, \mathbf{r}^{\prime} \mid \rho, \rho^{\prime}\right)$ in Eq. (7) is not explicitly translational invariant. Indeed, it is necessary the elaborate calculation in Sec. III to show that the function $\Gamma_{\mu v}\left(\mathbf{r}-\mathbf{r}^{\prime} ; \varepsilon, \omega\right)$ in Eq. (6) depends only on the difference of the coordinates.

The impurity-averaged quantities are obtained through standard diagrammatic methods. ${ }^{9}$ For $\Pi_{\varepsilon \omega}\left(\mathbf{r}, \mathbf{r}^{\prime} \mid \rho, \rho^{\prime}\right)$ we only consider the multiply crossed diagrams in Fig. 1(a) as being the relevant contribution to the conductivity in the weak localization regime. The averaged one-particle Green's function is given to lowest order by the diagram in Fig. 1(b):

$$
\begin{aligned}
G_{\varepsilon}\left(\mathbf{r}, \mathbf{r}^{\prime}\right)=G_{\varepsilon}^{0}\left(\mathbf{r}, \mathbf{r}^{\prime}\right)+U \int & d \mathbf{r}_{1} G_{\varepsilon}^{0}\left(\mathbf{r}, \mathbf{r}_{1}\right) \\
& \times G_{\varepsilon}^{0}\left(\mathbf{r}_{1}, \mathbf{r}_{1}\right) G_{\varepsilon}\left(\mathbf{r}_{1}, \mathbf{r}^{\prime}\right),
\end{aligned}
$$

where

$$
\begin{aligned}
G_{\varepsilon}^{0}\left(\mathbf{r}, \mathbf{r}^{\prime}\right)= & \sum_{\{\lambda\}} \psi_{\lambda}^{*}(\mathbf{r}) \psi_{\lambda}\left(\mathbf{r}^{\prime}\right) \\
& \times\left[\omega_{c}\left(n+\frac{1}{2}\right)+\frac{1}{2} k_{z}^{2}-\varepsilon_{F}-\varepsilon-i \eta \operatorname{sgn} \varepsilon\right]^{-1}
\end{aligned}
$$

and $\{\lambda\}=\left(n, k_{x}, k_{z}\right)$ indicates the set of Landau quantum numbers, while $\psi_{\lambda}(\mathbf{r})$ is the wave function for an electron in a magnetic field:

$$
\psi_{\lambda}(\mathbf{r})=\frac{e^{i\left(x k_{x}+z k_{z}\right)}}{2 \pi} \phi_{n}\left(y+k_{x} / \omega_{c}\right)
$$

$$
\phi_{n}(y)=\left[\frac{\omega_{c}}{\pi}\right]^{1 / 4}\left(2^{n} n !\right)^{-1 / 2} e^{-\omega_{c} y^{2} / 2} H_{n}\left(\left(\omega_{c}\right)^{1 / 2} y\right)
$$

Here $\omega_{c}=e B$ is the cyclotron frequency and $H_{n}(z)$ indicates a Hermite polynomial. An alternative expression for the Green's function in Eq. (10) is obtained by performing the integral over $k_{x}$, with the result

$G_{\varepsilon}^{0}\left(\mathbf{r}, \mathbf{r}^{\prime}\right)=\exp \left[i \frac{\omega_{c}}{2}\left(y+y^{\prime}\right)\left(x-x^{\prime}\right)\right] g_{\varepsilon}^{0}\left(\mathbf{r}-\mathbf{r}^{\prime}\right)$.

An explicit expression for $g_{\varepsilon}^{0}\left(\mathbf{r}-\mathbf{r}^{\prime}\right)$ is given below, but we find that $G_{\varepsilon}^{0}(r, r)$ is indeed independent of $r$, which allows us to solve for $G_{\varepsilon}\left(r, \mathbf{r}^{\prime}\right)$ in Eq. (9):

$$
\begin{aligned}
G_{\varepsilon}\left(\mathbf{r}, \mathbf{r}^{\prime}\right) & =\sum_{\{\lambda\}} \psi_{\lambda}^{*}(\mathbf{r}) \psi_{\lambda}\left(\mathbf{r}^{\prime}\right) G_{\varepsilon}\left(n, k_{z}\right) \\
& =\exp \left[i \frac{\omega_{c}}{2}\left(y+y^{\prime}\right)\left(x-x^{\prime}\right)\right] g_{\varepsilon}\left(\mathbf{r}-\mathbf{r}^{\prime}\right),
\end{aligned}
$$

with

$$
G_{\varepsilon}(n, \mathbf{p})=\left[\omega_{c}\left(n+\frac{1}{2}\right)+\frac{1}{2} p^{2}-\varepsilon_{F}-\varepsilon-\frac{i}{2 \tau} \operatorname{sgn} \varepsilon\right]^{-1},
$$
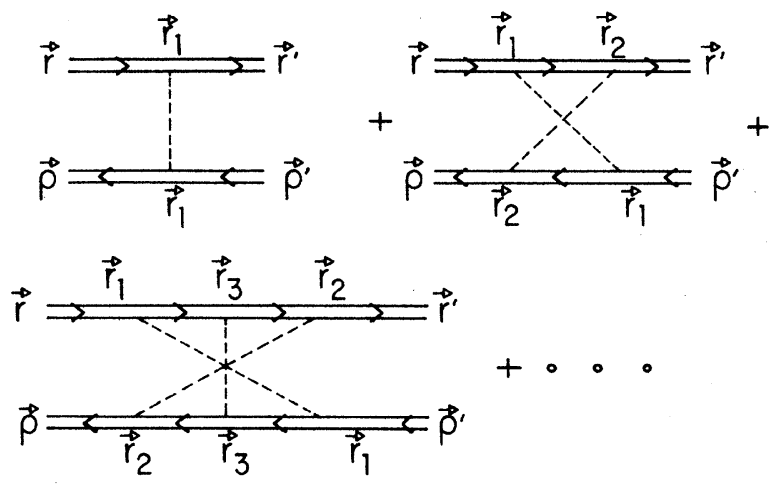

(a)

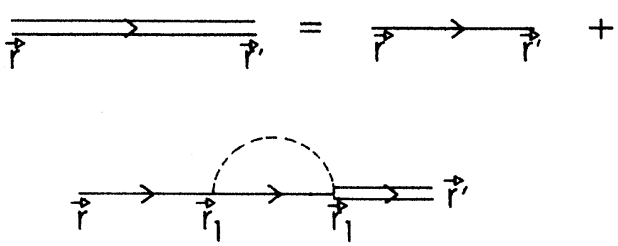

(b)

FIG. 1. (a) Diagrams that contribute to $\Pi_{\varepsilon+\omega, \omega}\left(\mathbf{r}, \mathbf{r}^{\prime} \mid \boldsymbol{\rho}, \boldsymbol{\rho}^{\prime}\right)$. Double solid lines indicate the propagator of (b) and pointed lines indicate the averaged impurity potential. (b) One-particle propagator $G_{\omega}\left(\mathbf{r} ; \mathbf{r}^{\prime}\right)$. 
$\frac{1}{2 \tau}=U \pi \omega_{c} \sum_{n=0}^{\infty} \int_{-\infty}^{\infty} d p \operatorname{Im} G_{\varepsilon}^{0}(n, \mathbf{p})=U 2 \pi\left(2 \varepsilon_{F}\right)^{1 / 2}$,

and

$$
\begin{aligned}
g_{\varepsilon}(\mathbf{r})=\omega_{c} \sum_{n} \int & \frac{d p}{2 \pi} e^{-i p z} e^{-\omega_{c}\left(x^{2}+y^{2}\right) / 4} \\
& \times L_{n}\left(\frac{1}{2} \omega_{c}\left(x^{2}+y^{2}\right)\right) G_{\varepsilon}(n, \mathbf{p}) .
\end{aligned}
$$

In the Green's function of Eq. (14) the Landau levels are considered exactly and it should be compared with the semiclassical expression used currently in the literature, ${ }^{5}$ where $g_{\varepsilon}(\mathbf{r})$ is replaced by the Green's function in the absence of a field, $g_{\varepsilon}(r, H=0)$. We will show in the following that it is precisely the use of the exact expression in Eq. (14) that gives rise to the anisotropic conductivity tensor.

The contribution to the polarization of the multiply crossed diagrams in Fig. 1(a) is

$$
\Pi_{\varepsilon, \omega}\left(\mathbf{r}, \mathbf{r}^{\prime} \mid \rho, \boldsymbol{\rho}^{\prime}\right)=G_{\varepsilon+\omega}\left(\mathbf{r} ; \mathbf{r}^{\prime}\right) G_{\varepsilon}\left(\boldsymbol{\rho}^{\prime} ; \boldsymbol{\rho}\right)+U \int d \mathbf{r}_{1} \int d \mathbf{r}_{2} G_{\varepsilon+\omega}\left(\mathbf{r} ; \mathbf{r}_{1}\right) G_{\varepsilon}\left(\boldsymbol{\rho}^{\prime}, \mathbf{r}_{1}\right) \widetilde{K}\left(\mathbf{r}_{1} ; \mathbf{r}_{2}\right) G_{\varepsilon+\omega}\left(\mathbf{r}_{2}, \mathbf{r}^{\prime}\right) G_{\varepsilon}\left(\mathbf{r}_{2}, \boldsymbol{\rho}\right)
$$

where

$$
\widetilde{K}\left(\mathbf{r}_{1} ; \mathbf{r}_{2}\right)=\delta\left(\mathbf{r}_{1}-\mathbf{r}_{2}\right)+U K\left(\mathbf{r}_{1} ; \mathbf{r}_{2}\right),
$$

$K\left(\mathbf{r}_{1} ; \mathbf{r}_{2}\right)$ is the solution of the integral equation

$$
\begin{aligned}
K\left(\mathbf{r}_{1} ; \mathbf{r}_{2}\right)= & K_{0}\left(\mathbf{r}_{1} ; \mathbf{r}_{2}\right) \\
& +U \int d \mathbf{r}_{3} K_{0}\left(\mathbf{r}_{1}, \mathbf{r}_{1}-\mathbf{r}_{3}\right) K\left(\mathbf{r}_{1}-\mathbf{r}_{3}, \mathbf{r}_{2}\right),
\end{aligned}
$$

and from Eq. (14),

$$
\begin{aligned}
K_{0}\left(\mathbf{r}, \mathbf{r}^{\prime}\right) & =G_{\varepsilon+\omega}\left(\mathbf{r}, \mathbf{r}^{\prime}\right) G_{\varepsilon}\left(\mathbf{r}, \mathbf{r}^{\prime}\right) \\
& =e^{i \omega_{c}\left(x-x^{\prime}\right)\left(y+y^{\prime}\right)} \Lambda_{0}\left(\mathbf{r}-\mathbf{r}^{\prime}\right) .
\end{aligned}
$$

The function

$$
\Lambda_{0}\left(\mathbf{r}-\mathbf{r}^{\prime}\right)=g_{\varepsilon+\omega}\left(\mathbf{r}-\mathbf{r}^{\prime}\right) g_{\varepsilon}\left(\mathbf{r}-\mathbf{r}^{\prime}\right)
$$

is related to the bare polarization loop of Fig. 2(a):

$$
\begin{aligned}
\Pi_{0}\left(\mathbf{r}-\mathbf{r}^{\prime}\right) & =G_{\varepsilon+\omega}\left(\mathbf{r}, \mathbf{r}^{\prime}\right) G_{\varepsilon}\left(\mathbf{r}^{\prime}, \mathbf{r}\right) \\
& =g_{\varepsilon+\omega}\left(\mathbf{r}-\mathbf{r}^{\prime}\right) g_{\varepsilon}\left(\mathbf{r}^{\prime}-\mathbf{r}^{\prime}\right) .
\end{aligned}
$$

The translational invariance of $\Pi_{0}\left(\mathbf{r}-\mathbf{r}^{\prime}\right)$ due to the phase cancellation in the Green's functions is not a trivial result and it was first obtained in two dimensions in Ref. 7 through a much laborious method.

The integral equation (20) cannot be solved trivially by a Fourier transformation due to the lack of translational invariance of $K_{0}\left(\mathbf{r}, \mathbf{r}^{\prime}\right)$ in Eq. (21), but we find that the solution can be written in a similar way:

$$
K\left(\mathbf{r}, \mathbf{r}^{\prime}\right)=e^{i \omega_{c}\left(\mathbf{x}-\mathbf{x}^{\prime}\right)\left(\mathbf{y}+\mathbf{y}^{\prime}\right)} \Lambda\left(\mathbf{r}-\mathbf{r}^{\prime}\right),
$$

where $\Lambda(\mathbf{r})$ satisfies the equation

$$
\begin{aligned}
\Lambda(\mathbf{r})=\Lambda_{0}(\mathbf{r})+U \int & d \mathbf{r}_{1} \Lambda_{0}\left(\mathbf{r}_{1}\right) \\
& \times e^{i \omega_{c}\left(x_{1} y-y_{1} x\right)} \Lambda\left(\mathbf{r}-\mathbf{r}_{1}\right) .
\end{aligned}
$$

Again the equation (24) does not separate in Fourier space due to the exponential in the integrand, but we find, by first expanding in powers of $\omega_{c}$ and later taking the Fourier transforms to lowest order in $\omega_{c}$,

$$
\begin{aligned}
\Lambda(\mathbf{k})=\bar{\Lambda}(\mathbf{k})+\frac{U}{1-U \Lambda_{0}(\mathbf{k})}\left[i \omega_{c}\left(\frac{\partial \Lambda_{0}}{\partial k_{x}} \frac{\partial \Lambda}{\partial k_{y}}-\frac{\partial \Lambda_{0}}{\partial k_{y}} \frac{\partial \Lambda}{\partial k_{x}}\right)\right. \\
\left.-\frac{1}{2} \omega_{c}^{2}\left(\frac{\partial^{2} \Lambda_{0}}{\partial k_{x}^{2}} \frac{\partial^{2} \Lambda}{\partial k_{y}^{2}}+\frac{\partial^{2} \Lambda_{0}}{\partial k_{y}^{2}} \frac{\partial^{2} \Lambda}{\partial k_{x}^{2}}-2 \frac{\partial^{2} \Lambda_{0}}{\partial k_{y} \partial k_{x}} \frac{\partial^{2} \Lambda}{\partial k_{y} \partial k_{x}}\right)+O\left(\omega_{c}^{3}\right)\right],
\end{aligned}
$$

where

$$
\bar{\Lambda}(\mathbf{k})=\frac{\Lambda_{0}(\mathbf{k})}{1-U \Lambda_{0}(\mathbf{k})}
$$

and $\Lambda_{0}(\mathbf{k})$ is the Fourier transform of $\Lambda_{0}(\mathbf{r})$ in Eq. (22). It is interesting to see that while $K\left(\mathbf{r}, \mathbf{r}^{\prime}\right)$ sums the multiply crossed diagrams of Fig. $1(\mathrm{a}), \bar{\Lambda}\left(\mathbf{r}-\mathbf{r}^{\prime}\right)$ sums the ladder diagrams of Fig. 2(b), in the presence of a magnetic field. In this last case the phases cancel between the propagators going in opposite directions from Eq. (14) and the integral equation for $\Lambda(r)$ would be similar to that in Eq. (24) without the exponential in the integrand.

Before solving for $\Lambda(\mathbf{k})$ in Eq. (25) we must calculate, from Eqs. (22) and (17),

$$
\begin{aligned}
\Lambda_{0}(\mathbf{k})=e^{\omega_{c} k_{\perp}^{2} / 2} \int d p \omega_{c} \sum_{n, n^{\prime}} & G_{\varepsilon+\omega^{\prime}}\left(n, p+k_{z}\right) G_{\varepsilon}\left(n^{\prime}, p\right) \\
& \times(-1)^{n+n^{\prime}} L_{n}^{n-n^{\prime}}\left(k_{\perp}^{2} / 2 \omega_{c}\right) \\
& \times L_{n^{\prime}}^{n^{\prime}-n}\left(k_{\perp}^{2} / 2 \omega_{c}\right)
\end{aligned}
$$

where $k_{\perp}^{2}=k_{x}^{2}+k_{y}^{2}$ is the momentum on the plane perpendicular to the field and $L_{n}^{m}(z)$ are the Laguerre polynomials. We find upon expanding Eq. (27) to lowest order in momentum, in the limit $\varepsilon+\omega=0^{+}, \varepsilon=0^{-}$, and to leading order in $1 / \tau \varepsilon_{F}<1$ :

$$
\Lambda_{0}(\mathbf{k})=C_{0}-C_{z} k_{z}^{2}-C_{\perp} k_{\perp}^{2}
$$



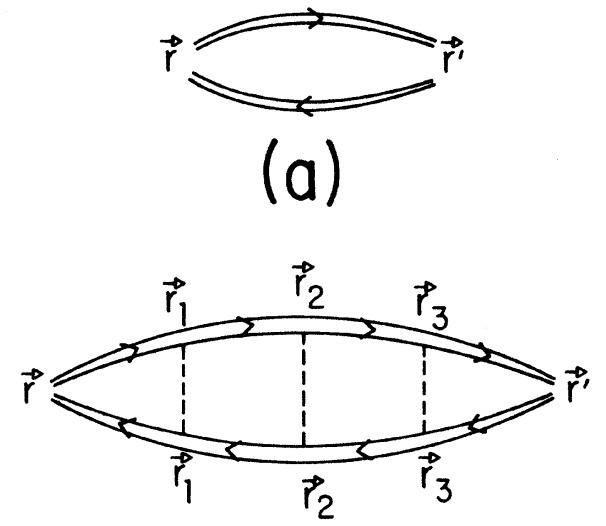

(b)

FIG. 2. (a) Bare polarization loop. (b) Ladder diagram for the polarization.

with

$$
\begin{aligned}
C_{0} & =\omega_{c} \sum_{n} \int_{-\infty}^{\infty} d p G_{\varepsilon+\omega}(n, p) G_{\varepsilon}(n, p) \\
& =\pi \tau 2 \sqrt{2} \frac{1}{1-i \omega \tau} \omega_{c} \sum_{n} \operatorname{Re}\left[\varepsilon_{F}-\omega_{c} n+\frac{i}{2 \tau}\right]^{-1 / 2}, \\
C_{z} & =\omega_{c} \sum_{n} \int_{-\infty}^{\infty} d p G_{\varepsilon+\omega}(n, p)\left\{\frac{1}{2}\left[G_{\varepsilon}(n, p)\right]^{2}\right. \\
& \left.\left.=\pi 4 \sqrt{2} \tau^{3} \omega_{c} \sum_{n} \operatorname{Re}\left[\varepsilon_{F}-\omega_{c} n+i / 2 \tau\right]_{\varepsilon}(n, p)\right]^{3}\right\} \\
C_{\perp} & =\operatorname{Re}\left[\omega _ { c } ^ { 2 } \sum _ { n } \int _ { d p } G _ { \varepsilon + \omega } ( n , p ) \left[\left(n+\frac{1}{2}\right) G_{\varepsilon}(n, p)\right.\right. \\
& =\frac{\pi 2 \sqrt{2} \tau^{3}}{1+\omega_{c}^{2} \tau^{2}} \omega_{c}^{2} \sum_{n} n \operatorname{Re}\left[\varepsilon_{F}-\omega_{c} n+\frac{i}{2 \tau}\right]^{-1 / 2}
\end{aligned}
$$

In the limit $\omega_{c} / \varepsilon_{F}, 1 / \tau \varepsilon_{F} \ll 1$, we have

$$
\begin{aligned}
{\left[\varepsilon_{F}-\omega_{c} n+\frac{i}{2 \tau}\right]^{\alpha} \approx } & \Theta\left(\varepsilon_{F} / \omega_{c}-n\right)\left(\varepsilon_{F}-\omega_{c} n\right)^{\alpha} \\
& +\Theta\left(n-\varepsilon_{F} / \omega_{c}\right) e^{+i \pi \alpha}\left(\omega_{c} n-\varepsilon_{F}\right)^{\alpha},
\end{aligned}
$$

and the sums over $n$ can be performed by using, ${ }^{10}$ for $N=\varepsilon_{F} / \omega_{c}$,

$$
\sum_{n=1}^{N} n^{\alpha}=\frac{N^{\alpha+1}}{\alpha+1}\left[1+\frac{1}{2} \frac{\alpha+1}{N}+O\left(1 / N^{2}\right)\right] .
$$

The leading term in Eq. (33) corresponds to replacing the sum by an integral and the first correction is $O\left(\omega_{c} / \varepsilon_{F}\right)$. We then have in Eq. (28)

$$
\Lambda_{0}(\mathbf{k})=\frac{1}{U}\left(1+i \omega \tau-D_{0} \tau k_{z}^{2}-D_{H} \tau k_{\perp}^{2}\right),
$$

where we used Eq. (16) for $\tau$, and $D_{0}=2 \varepsilon_{F} \tau / 3$ is the three-dimensional diffusion coefficient, while $D_{H}=D_{0}(1$ $\left.+\omega_{c}^{2} \tau^{2}\right)^{-1}$. The two-dimensional result for $\Pi_{0}(\mathbf{k})$ in Ref. (7) is obtained from Eq. (34) by eliminating $k_{z}^{2}$ and replacing $D_{0}$ by its two-dimensional expression.

Going back to Eq. (25), we solve it to $O\left(\omega_{c}^{2}\right)$ by approximating $\Lambda(k) \approx \bar{\Lambda}(\mathbf{k})$ at the right-hand side. The linear term in $\omega_{c}$ will then vanish, and we obtain from Eq. (34)

$$
\begin{aligned}
1+U \Lambda(\mathbf{k}) & \approx \frac{1}{1-U \Lambda_{0}(\mathbf{k})}\left[1-\frac{1}{2} \omega_{c}^{2}\left(\frac{D_{H} \tau}{1-U \Lambda_{0}(\mathbf{k})}\right]^{2}\right] \\
& \approx \frac{D_{0} \tau k_{z}^{2}+D_{H} \tau k_{\perp}^{2}}{\left(D_{0} \tau k_{z}^{2}+D_{H} \tau k_{\perp}^{2}\right)^{2}+\frac{1}{2}\left(\omega_{c} D_{0} \tau\right)^{2}}
\end{aligned}
$$

From Eqs. (23) and (35) we have in Eq. (19):

$$
\begin{aligned}
\widetilde{K}\left(\mathbf{r}, \mathbf{r}^{\prime}\right)= & e^{i \omega_{c}\left(x-x^{\prime}\right)\left(y+y^{\prime}\right)} \\
& \times \int \frac{d \mathbf{k}}{(2 \pi)^{3}} e^{i \mathbf{k} \cdot\left(\mathbf{r}-\mathbf{r}^{\prime}\right)}[1+U \Lambda(\mathbf{k})]
\end{aligned}
$$

If we compare Eq. (36) with the usual expression for the "diffusion propagator" in the presence of a field quoted by other authors, ${ }^{4-6}$ that is,

$$
U K_{D}\left(\mathbf{r}, \mathbf{r}^{\prime}\right)=\sum_{\{\lambda\}} \frac{\psi_{\lambda}^{*}(\mathbf{r}) \psi_{\lambda}\left(\mathbf{r}^{\prime}\right)}{D_{0} \tau\left[\omega_{c}^{*}\left(n+\frac{1}{2}\right)+\frac{1}{2} k_{z}^{2}\right]},
$$

where $\omega_{c}^{*}$ is the cyclotron frequency for a particle with charge $e^{*}=2 e$ and $\psi_{\lambda}(\mathbf{r})$ are the eigenfunctions of Eq. (11) with $\omega_{c}$ replaced by $\omega_{c}^{*}$, we see that both expressions do not agree. This is because Eqs. (36) and (37) involve different degrees of approximation: while Eqs. (23) and (24) are exact and the only approximation involved is the expansion of $\Lambda(\mathbf{k})$ to $O\left(k^{2}, \omega_{c}^{2}\right)$ in Eq. (35), the derivation of Eq. (37) is of a more phenomenological kind. It is obtained by considering the sum of diagrams in Fig. 1(a) without a magnetic field and in the diffusion pole approximation as a propagator of a single particle of double charge $e^{*}=2 e$ and momentum q; afterwards, the magnetic field is switched on by making the replacement $\mathbf{q} \rightarrow$ [eigenvalue of $\left.\left(-i \boldsymbol{\nabla}-e^{*} \mathbf{A}_{0}\right)\right]$.

On general symmetry arguments we can see that Eq. (35) has the correct dependence on $\omega_{c}^{2}$, as the diagonal components of the conductivity tensor have to be invariant under the transformation $B \rightarrow-B$.

\section{CALCULATION OF $\sigma_{x x}=\sigma_{y y}$ AND $\sigma_{z z}$}

To obtain an explicit expression for the conductivity, we make in Eq. (36) the standard approximation ${ }^{5}$

$\widetilde{K}\left(\mathbf{r}, \mathbf{r}^{\prime}\right) \approx e^{i \omega_{c}\left(x-x^{\prime}\right)\left(y+y^{\prime}\right)} \widetilde{K}(0)$, 


$$
\begin{aligned}
\widetilde{K}(0)=\frac{1}{(2 \pi)^{3}} \int d k_{z} \int d \mathbf{k}_{\perp} \\
\quad \times \frac{D_{0} \tau k_{z}^{2}+D_{H} \tau k_{\perp}^{2}}{\left(D_{0} \tau k_{z}^{2}+D_{H} \tau k_{\perp}^{2}\right)^{2}+\frac{1}{2}\left(\omega_{c} D_{0} \tau\right)^{2}}
\end{aligned}
$$

because we consider that the main contribution to the integral will come from the diffusion pole at $k^{2} \approx 0$. We obtain from Eq. (18)

$$
\Pi_{\varepsilon, \omega}\left(\mathbf{r}, \mathbf{r}^{\prime} \mid \boldsymbol{\rho}, \boldsymbol{\rho}^{\prime}\right)=G_{\varepsilon+\omega}\left(\mathbf{r}, \mathbf{r}^{\prime}\right) G_{\varepsilon}\left(\boldsymbol{\rho}^{\prime}, \boldsymbol{\rho}\right)+\Pi_{\varepsilon, \omega}^{\prime}\left(\mathbf{r}, \mathbf{r}^{\prime} \mid \boldsymbol{\rho}, \boldsymbol{\rho}^{\prime}\right),
$$

$$
\begin{aligned}
\Pi_{\varepsilon, \omega}^{\prime}\left(\mathbf{r}, \mathbf{r}^{\prime} \mid \boldsymbol{\rho}, \boldsymbol{\rho}^{\prime}\right) & \\
=U \widetilde{K}(0) \int d \mathbf{r}_{1} \int & d \mathbf{r}_{2} G_{\varepsilon+\omega}\left(\mathbf{r}, \mathbf{r}_{1}\right) G_{\varepsilon}\left(\boldsymbol{\rho}^{\prime}, \mathbf{r}_{1}\right) \\
& \times e^{i \omega_{c}\left(x_{1}-x_{2}\right)\left(y_{1}+y_{2}\right)} \\
& \times G_{\varepsilon+\omega}\left(\mathbf{r}_{2}, \mathbf{r}^{\prime}\right) G_{\varepsilon}\left(\mathbf{r}_{2}, \boldsymbol{\rho}\right) .
\end{aligned}
$$

The first term on the right-hand side of Eq. (40) gives the Lorentz contribution $\sigma_{\mu \nu}^{0}$. We then write the conductivity tensor:

$$
\sigma_{\mu \nu}(\mathbf{q}, \omega)=\sigma_{\mu \nu}^{0}(\mathbf{q}, \omega)+\sigma_{\mu \nu}^{\prime}(\mathbf{q}, \omega),
$$

where the anomalous contribution $\sigma_{\mu \nu}^{\prime}(\mathbf{q}, \omega)$ is calculated by introducing Eq. (41) in Eq. (6).

The detailed calculations are shown in the Appendix, and we obtain for the dc components of the tensor:

$$
\begin{aligned}
& \sigma_{z z}^{\prime}=-\frac{e^{2}}{2 \pi^{2}} U \widetilde{K}(0) \int_{-\infty}^{\infty} \frac{d p}{2 \pi} \omega_{c} \sum_{n=0}^{\infty} p^{2}\left[G_{+}(n, p) G_{-}(n, p)\right]^{2}, \\
& \sigma_{\perp}^{\prime}=-\frac{e^{2}}{2 \pi^{2}} U \widetilde{K}(0) \int_{-\infty}^{\infty} \frac{d p}{2 \pi} \omega_{c}^{2} \sum_{n=0}^{\infty}\left[\frac{n+1}{2}\left[G_{+}(n, p) G_{-}(n+1, p)\right]^{2}+\frac{n}{2}\left[G_{+}(n, p) G_{-}(n-1, p)\right]^{2}\right),
\end{aligned}
$$

where now $\sigma_{\perp}^{\prime}=\sigma_{y y}^{\prime}=\sigma_{x x}^{\prime}$, and

$$
G_{ \pm}(n, p)=\left[\omega_{c}\left(n+\frac{1}{2}\right)+\frac{1}{2} p^{2}-\mu \pm \frac{i}{2 \tau}\right]^{-1} \text {. }
$$

From Eq. (44) and Eq. (45) we can also write

$$
\begin{aligned}
\sigma_{\perp}^{\prime}=- & \frac{e^{2}}{2 \pi^{2}} U \widetilde{K}(0) \\
& \times \operatorname{Re}\left[\int_{-\infty}^{\infty} \frac{d p}{2 \pi} \omega_{c}^{2} \sum_{n=0}^{\infty} n\left[G_{+}(n, p) G_{-}(n+1, p)\right]^{2}\right] .
\end{aligned}
$$

It is clear from Eq. (43) and Eq. (46) that the diagonal components of the conductivity tensor are anisotropic, as expected. What remains to be calculated is for which values of the applied field the anisotropic effect should be taken into account, in order to compare with the isotropic predictions of other theories. ${ }^{1,2}$

We obtain for $\widetilde{K}(0)$ from Eq. (39)

$$
\begin{aligned}
& \widetilde{K}(0)=\frac{1}{2 \pi^{2}} \frac{1+\omega_{c}^{2} \tau^{2}}{\left(D_{0} \tau\right)^{3 / 2}} I_{0}, \\
& I_{0}=\int_{0}^{\infty} d p \frac{p^{4}}{p^{4}+\frac{1}{2}\left(\omega_{c} D_{0} \tau\right)^{2}} \\
& =I^{*}-\pi 2^{-3 / 4}\left(\omega_{c} D_{0} \tau\right)^{1 / 2},
\end{aligned}
$$

where $I^{*}$ is a constant, independent of the applied field. That this constant, is in fact infinite is spurious result, due to the approximation in Eq. (39). The sums over $n$ in Eqs. (43) and (46) are resolved in the approximations of Eqs. (32) and (33), with the result:

$$
\begin{gathered}
\int_{-\infty}^{\infty} \frac{d p}{2 \pi} \omega_{c} \sum_{n=0}^{\infty} p^{2}\left[G_{+}(n, p) G_{-}(n, p)\right]^{2} \\
=4 \sqrt{3}\left(D_{0} \tau\right)^{3 / 2}\left[1+O\left(\omega_{c} / G_{F}, 1 / G_{F} \tau\right)\right] \\
\operatorname{Re}\left[\int_{-\infty}^{\infty} \frac{d p}{2 \pi} \omega_{c}^{2} \sum_{n-0}^{\infty} n\left[G_{+}(n, p) G_{-}(n+1, p)\right]^{2}\right] \\
=4 \sqrt{3}\left(D_{0} \tau\right)^{3 / 2}\left[\frac{1-3 \omega_{c}^{2} \tau^{2}}{\left(1+\omega_{c}^{2} \tau^{2}\right)^{3}}+O\left(\omega_{c} / G_{F}, 1 / G_{F} \tau\right)\right]
\end{gathered}
$$

We get, by introducing Eqs. (47), (48), and (49) into Eqs. (43) and (46),

$$
\begin{aligned}
& \sigma_{z z}^{\prime}\left(\omega_{c}\right)-\sigma_{z z}^{\prime}(0) \approx e^{2} U / \pi^{4}\left[\pi 2^{-3 / 4}\left(\omega_{c} D_{0} \tau\right)^{1 / 2}\right. \\
& \left.-I^{*}\left(\omega_{c} \tau\right)^{2}\right], \\
& \sigma_{\perp}^{\prime}\left(\omega_{c}\right)-\sigma_{\perp}^{\prime}(0) \approx e^{2} U / \pi^{4}\left[\pi 2^{-3 / 4}\left(\omega_{c} D_{0} \tau\right)^{1 / 2}\right. \\
& \left.+5 I^{*}\left(\omega_{c} \tau\right)^{2}\right] \text {. }
\end{aligned}
$$

It is interesting to see that the anisotropic contribution of $O\left(\left(\omega_{c} \tau\right)^{2}\right)$ goes in the direction of increasing the transverse conductivity $\sigma_{\perp}^{\prime}$, thus decreasing the localization effect, as expected. However, in the component parallel to the field, $\sigma_{z z}^{\prime}$, this term goes in the opposite direction. It is also clear from Eqs. (50) and (51) that the anisotropy will not be seen in the "high-field" limit usually considered, ${ }^{1}$ that is, $\left(D_{0} \tau_{i}\right)^{-1}<\omega_{c}<\left(D_{0} \tau\right)^{-1}<<1$, where $\tau_{i}$ is the inelastic lifetime, ${ }^{5,6}$ thus explaining the agreement of the isotropic calculation with the experimental data.

A word should be said about the absence of the inelastic lifetime in our results. While in the two-dimensional calculations at zero field the integral in Eq. (39) would diverge in the infrared, thus making it necessary to intro- 
duce phenomenologically a lower cutoff $k_{\perp}^{2} \approx \tau_{i}^{-1}$, the same integral in three dimensions is infrared convergent, and we considered the introduction of $\tau_{i}$ to be artificial.

Technically, the inelastic lifetime is introduced ${ }^{5}$ by replacing $i \omega$ by $\tau_{i}^{-1}$ in Eq. (34), while in all other Green's functions the frequency is allowed to vanish.

\section{CONCLUSIONS}

In this paper we studied the three-dimensional magnetoconductance in the weak localization regime, within a formalism based from the start on the Landau eigenfunctions and avoiding the semiclassical approximation used in previous calculations. ${ }^{1,2,5,6}$ Our results predict anisotropic diagonal components of the conductivity tensor, although the anisotropy terms only become relevant for very high fields $\omega_{c} \tau>1$, while in the range $\omega_{c} \tau<D_{0}^{-1}<1$ usually considered in the literature ${ }^{1}$ we recover the isotropic behavior in $\left(\omega_{c}\right)^{1 / 2}$ predicted by Kawabata. ${ }^{2}$

\section{ACKNOWLEDGMENTS}

This work was supported in part by Conselho Nacional de Desenvolvimento Cientifico e Tecnològico (CNPq) and in part by Financiadora de Estudos e Projetos (FINEP), Brazil.

\section{APPENDIX}

To calculate Eq. (41) it is more convenient to write from Eq. (14)

$\Pi_{\varepsilon, \omega}^{\prime}\left(\mathbf{r}, \mathbf{r}^{\prime} \mid \rho, \rho^{\prime}\right)=U \widetilde{K}(0) \sum_{\left\{\lambda_{i}\right\}}\left[\psi_{\lambda_{1}}^{*}(\mathbf{r}) \psi_{\lambda_{2}}^{*}\left(\boldsymbol{\rho}^{\prime}\right) \psi_{\lambda_{3}}\left(\mathbf{r}^{\prime}\right) \psi_{\lambda_{4}}(\boldsymbol{\rho}) I\left(\lambda_{1}, \lambda_{2}, \lambda_{3}, \lambda_{4}\right) G_{+}\left(n_{1}, k_{1 z}\right) G_{-}\left(n_{2}, k_{2 z}\right) G_{+}\left(n_{3}, k_{3 z}\right) G_{-}\left(n_{4}, k_{4 z}\right)\right]$

where

$$
\begin{aligned}
& G_{+}(n, p)=G_{\varepsilon+\omega}(n, p), \quad G_{-}(n, p)=G_{\varepsilon}(n, p) \\
& \begin{aligned}
I\left(\lambda_{1}, \lambda_{2}, \lambda_{3}, \lambda_{4}\right)= & \int d \mathbf{r}_{1} \int d \mathbf{r}_{2} e^{i \omega_{c}\left(x_{1}-x_{2}\right)\left(y_{1}+y_{2}\right)} \psi_{\lambda_{1}}\left(\mathbf{r}_{1}\right) \psi_{\lambda_{2}}\left(\mathbf{r}_{1}\right) \psi_{\lambda_{3}}^{*}\left(\mathbf{r}_{2}\right) \psi_{\lambda_{4}}^{*}\left(\mathbf{r}_{2}\right) \\
= & (2 \pi)^{4} / \omega_{c} \delta\left(k_{1 z}+k_{2 z}\right) \delta\left(k_{3 z}+k_{4 z}\right) \delta\left(k_{1 x}+k_{2 x}-k_{3 x}-k_{4 x}\right)(-1)^{n_{3}+n_{4}} \\
& \times \int_{-\infty}^{\infty} d y_{1} \phi_{n_{1}}\left(y_{1}+k_{1 x} / \omega_{c}\right) \phi_{n_{2}}\left(y_{1}+k_{2 x} / \omega_{c}\right) \phi_{n_{3}}\left(y_{1}+k_{4 x} / \omega_{c}\right) \phi_{n_{4}}\left(y_{1}+k_{3 x} / \omega_{c}\right)
\end{aligned}
\end{aligned}
$$

Introducing then Eq. (A1) into Eq. (6), we obtain for the diagonal components:

$$
\begin{aligned}
\Gamma_{\mu \mu}^{\prime}\left(\mathbf{r}, \mathbf{r}^{\prime}, \varepsilon, \omega\right)=U \widetilde{K}(0) \frac{2 \pi}{\omega_{c}} \sum_{\substack{n_{1}, n_{2} \\
n_{3}, n_{4}}} \int D k \int & d y_{1} F_{\mu \mu}\left(\mathbf{r}, \mathbf{r}^{\prime}, k_{i}, n_{i}\right) e^{-i\left[\left(k_{1 z}+k_{3 z}\right)\left(r_{z}-r_{z}^{\prime}\right)+k_{x}\left(r_{x}-r_{x}^{\prime}\right)\right]} \\
& \times G_{-}\left(n_{2}, k_{1 z}\right) G_{+}\left(n_{1}, k_{1 z}\right) G_{+}\left(n_{3}, k_{3 z}\right) G_{-}\left(n_{4}, k_{3 z}\right)(-1)^{n_{3}+n_{4}} \\
& \times \phi_{n_{1}}\left(y_{1}+k_{1 x} / \omega_{c}\right) \phi_{n_{2}}\left(y_{1}\left(k_{3 x}-k_{x}\right) / \omega_{c}\right) \\
& \times \phi_{n_{3}}\left(y_{1}+\left(k_{1 x}-k_{x}\right) / \omega_{c}\right) \phi_{n_{4}}\left(y_{1}+k_{3 x} / \omega_{c}\right)
\end{aligned}
$$

where

$$
D k \equiv \frac{1}{(2 \pi)^{5}} d k_{1 x} d k_{1 z} d k_{3 x} d k_{3 z} d k_{x}
$$

and

$$
\begin{aligned}
F_{z z}\left(\mathbf{r}, \mathbf{r}^{\prime} ; k_{i}, n_{i}\right)= & -\left(k_{1 z}-k_{3 z}\right)^{2} \phi_{n_{1}}\left(r_{y}+k_{1 x} / \omega_{c}\right) \phi_{n_{2}}\left(r_{y}^{\prime}+\left(k_{3 x}-k_{1 x}\right) / \omega_{c}\right) \phi_{n_{3}}\left(r_{y}^{\prime}+k_{3 x} / \omega_{c}\right) \phi_{n_{4}}\left(r_{y}+\left(k_{1 x}-k_{x}\right) / \omega_{c}\right), \\
F_{y y}\left(\mathbf{r}, \mathbf{r}^{\prime} ; k_{i}, n_{i}\right)= & \omega_{c}\left[\phi_{n_{1}}^{\prime}\left(r_{y}+k_{1 x} / \omega_{c}\right) \phi_{n_{4}}\left(r_{y}+\left(k_{1 x}-k_{x}\right) / \omega_{c}\right)-\phi_{n_{1}}\left(r_{y}+k_{1 x} / \omega_{c}\right) \phi_{n_{4}}^{\prime}\left(r_{y}+\left(k_{1 x}-k_{x}\right) / \omega_{c}\right)\right] \\
& \times\left[\phi_{n_{2}}^{\prime}\left(r_{y}^{\prime}+\left(k_{3 x}-k_{x}\right) / \omega_{c}\right) \phi_{n_{3}}\left(r_{y}^{\prime}+k_{3 x} / \omega_{c}\right)-\phi_{n_{2}}\left(r_{y}^{\prime}+\left(k_{3 x}-k_{x}\right) / \omega_{c}\right) \phi_{n_{3}}^{\prime}\left(r_{y}^{\prime}+k_{3 x} / \omega_{c}\right)\right], \\
F_{x x}\left(\mathbf{r}, \mathbf{r}^{\prime} ; k_{i}, n_{i}\right)= & \left(2 k_{1 x}-k_{x}+2 \omega_{c} r_{y}\right)\left(2 k_{3 x}-k_{x}+2 \omega_{c} r_{y}^{\prime}\right) \phi_{n_{1}}\left(r_{y}+k_{1 x} / \omega_{c}\right) \phi_{n_{2}}\left(r_{y}^{\prime}+\left(k_{3 x}-k_{1 x}\right) / \omega_{c}\right) \\
& \times \phi_{n_{3}}\left(r_{y}^{\prime}+k_{3 x} / \omega_{c}\right) \phi_{n_{4}}\left(r_{y}+\left(k_{1 x}-k_{x}\right) / \omega_{c}\right) .
\end{aligned}
$$

The translational invariance of Eq. (A4) appears explicitly if we make the change of variables 


$$
\begin{aligned}
& k_{1 x}=p_{1 x}-\frac{1}{2} \omega_{c}\left(r_{y}+r_{y}^{\prime}\right), \quad k_{1 z}=p_{1 z}, \\
& k_{3 x}=p_{3 x}-\frac{1}{2} \omega_{c}\left(r_{y}+r_{y}^{\prime}\right), \quad k_{3 z}=p_{3 z}, \\
& y_{1}=y+\frac{1}{2}\left(r_{y}+r_{y}^{\prime}\right)
\end{aligned}
$$

to obtain from Eqs. (A5) and (A6), for $\mathbf{R}=\left(\mathbf{r}-\mathbf{r}^{\prime}\right) / 2$,

$$
\begin{aligned}
F_{z z}\left(\mathbf{R}, p_{i}, n_{i}\right)= & \left.\left(p_{1 z}-p_{3 z}\right)^{2} \phi_{n_{1}}\left(R_{y}+p_{1 x} / \omega_{c}\right) \phi_{n_{2}}\left(-R_{y}+\left(p_{3 x}-p_{x}\right) / \omega_{c}\right)\right) \\
& \times \phi_{n_{3}}\left(-R_{y}+p_{3 x} / \omega_{c}\right) \phi_{n_{4}}\left(R_{y}+\left(p_{1 x}-p_{x}\right) / \omega_{c}\right), \\
F_{y y}\left(\mathbf{R}, p_{i}, n_{i}\right)= & \omega_{c}\left[\phi_{n_{1}}^{\prime}\left(R_{y}+p_{1 x} / \omega_{c}\right) \phi_{n_{4}}\left(R_{y}+\left(p_{1 x}-p_{x}\right) / \omega_{c}\right)-\phi_{n_{1}}\left(R_{y}+p_{1 x} / \omega_{c}\right) \phi_{n_{4}}^{\prime}\left(R_{y}+\left(p_{1 x}-p_{x}\right) / \omega_{c}\right)\right] \\
& \times\left[\phi_{n_{2}}^{\prime}\left(-R_{y}+\left(p_{3 x}-p_{x}\right) / \omega_{c}\right) \phi_{n_{3}}\left(-R_{y}+p_{3 x} / \omega_{c}\right)-\phi_{n_{2}}\left(-R_{y}+\left(p_{3 x}-p_{x}\right) / \omega_{c}\right) \phi_{n_{c}}^{\prime}\left(-R_{y}+p_{3 x} / \omega_{c}\right)\right],
\end{aligned}
$$

$$
\begin{aligned}
F_{x x}\left(\mathbf{R}, p_{i}, n_{i}\right)= & \left(2 p_{1 x}-p_{x}+2 \omega_{c} R_{y}\right)\left(2 p_{3 x}-p_{x}-2 \omega_{c} R_{y}\right) \phi_{n_{1}}\left(R_{y}+p_{1 x} / \omega_{c}\right) \phi_{n_{2}}\left(-R_{y}+\left(p_{3 x}-p_{x}\right) / \omega_{c}\right) \\
& \times \phi_{n_{3}}\left(-R_{y}+p_{3 x} / \omega_{c}\right) \phi_{n_{4}}\left(R_{y}+\left(p_{1 x}-p_{x}\right) / \omega_{c}\right)
\end{aligned}
$$

while the rest of the integrand in Eq. (A3) is left invariant. The calculation of $\Gamma_{\mu \mu}^{\prime}(\mathbf{R}, \varepsilon, \omega)$ cannot proceed further because each integration variable appears simultaneously in four Hermite polynomials and the integrals cannot be solved in closed form.

However, the calculation of the dc conductivity from Eq. (5),

$$
\sigma_{\mu \mu}^{\prime}(0,0)=\frac{e^{2}}{4 \pi} \int d \mathbf{R} \Gamma_{\mu \mu}^{\prime}\left(\mathbf{R} ; 0^{-}, 0^{+}\right)
$$

simplifies if we perform another change of variables:

$$
\begin{aligned}
& v_{1}=y+p_{1 x} / \omega_{c}, \quad v_{2}=y+p_{3 x} / \omega_{c}, \\
& v_{3}=R_{y}+p_{1 x} / \omega_{c}, \quad v_{4}=-R_{y}+p_{3 x} / \omega_{c},
\end{aligned}
$$

with Jacobian

$$
\frac{\partial\left(p_{1 x}, p_{3 x}, y, R_{y}\right)}{\partial\left(v_{1}, v_{2}, v_{3}, v_{4}\right)}=\omega_{c}^{2} .
$$

We obtain

$$
\begin{aligned}
& \sigma_{z z}^{\prime}=\frac{e^{2}}{2 \pi^{2}} U \widetilde{K}(0) \omega_{c} \int_{-\infty}^{\infty} \frac{d p}{2 \pi} \sum_{\substack{n_{1}, n_{2}, n_{3}, n_{4}}} p^{2} G_{+}\left(n_{1}, p\right) G_{-}\left(n_{2}, p\right) G_{+}\left(n_{3}, p\right) G_{-}\left(n_{4}, p\right)(-1)^{n_{3}+n_{4}} \\
& \times \int_{-\infty}^{\infty} d v_{1} \phi_{n_{1}}\left(v_{1}\right) \phi_{n_{3}}\left(v_{1}\right) \\
& \times \int_{-\infty}^{\infty} d v_{2} \phi_{n_{2}}\left(v_{2}\right) \phi_{n_{4}}\left(v_{2}\right) \int_{-\infty}^{\infty} d v_{3} \phi_{n_{1}}\left(v_{3}\right) \phi_{n_{4}}\left(v_{3}\right) \int_{-\infty}^{\infty} d v_{4} \phi_{n_{2}}\left(v_{4}\right) \phi_{n_{3}}\left(v_{4}\right) \text {, } \\
& \sigma_{y y}^{\prime}=\frac{e^{2}}{2 \pi^{2}} U \widetilde{K}(0) \omega_{c}^{2} \int_{-\infty}^{\infty} \frac{d p}{2 \pi} \sum_{\substack{n_{1}, n_{2}, n_{3}, n_{4}}} G_{+}\left(n_{1}, p\right) G_{-}\left(n_{2}, p\right) G_{+}\left(n_{3}, p\right) G_{-}\left(n_{4}, p\right)(-1)^{n_{3}+n_{4}} \\
& \times \int d v_{1} \phi_{n_{1}}\left(v_{1}\right) \phi_{n_{3}}\left(v_{1}\right) \\
& \times \int d v_{2} \phi_{n_{2}}\left(v_{2}\right) \phi_{n_{4}}\left(v_{2}\right) \int d v_{3} \phi_{n_{1}}^{\prime}\left(v_{3}\right) \phi_{n_{4}}\left(v_{3}\right) \int d v_{4} \phi_{n_{2}}^{\prime}\left(v_{4}\right) \phi_{n_{3}}\left(v_{4}\right) \text {, }
\end{aligned}
$$




$$
\begin{aligned}
\sigma_{x x}^{\prime}=\frac{e^{2}}{2 \pi^{2}} U \widetilde{K}(0) \omega_{c}^{2} \int_{-\infty}^{\infty} \frac{d p}{2 \pi} \sum_{\substack{n_{1}, n_{2}, n_{3}, n_{4}}} G_{+}\left(n_{1}, p\right) G_{-}\left(n_{2}, p\right) G_{+}\left(n_{3}, p\right) G_{-}\left(n_{4}, p\right)(-1)^{n_{3}+n_{4}} \\
\times \int d v_{1} \phi_{n_{1}}\left(v_{1}\right) \phi_{n_{3}}\left(v_{1}\right) \\
\times \int d v_{2} \phi_{n_{2}}\left(v_{2}\right) \phi_{n_{4}}\left(v_{2}\right) \int d v_{3} v_{3} \phi_{n_{1}}\left(v_{3}\right) \phi_{n_{4}}\left(v_{3}\right) \int d v_{4} v_{4} \phi_{n_{2}}\left(v_{4}\right) \phi_{n_{3}}\left(v_{4}\right),
\end{aligned}
$$

and using the relations

$$
\begin{aligned}
& \int_{-\infty}^{\infty} d v \phi_{n}(v) \phi_{m}(v)=\delta_{n, m}, \\
& \int_{-\infty}^{\infty} d v \phi_{n}^{\prime}(v) \phi_{m}(v)=\delta_{m, n-1}(n / 2)^{1 / 2}-\delta_{m, n+1}[(n+1) / 2]^{1 / 2}, \\
& \int_{-\infty}^{\infty} d v v \phi_{n}(v) \phi_{m}(v)=\delta_{m, n-1}\left[\frac{n}{2}\right)^{1 / 2}+\delta_{m, n+1}[(n+1) / 2]^{1 / 2},
\end{aligned}
$$

we get Eqs. (43)-(45).

${ }^{1}$ B. L. Altshuler, D. Khmel'nitzkii, A. I. Larkin, and P. A. Lee, Phys. Rev. B 22, 5142 (1980).

${ }^{2}$ A. Kawabata, Solid State Commun. 34, 431 (1980).

${ }^{3}$ E. Abrahams, P. W. Anderson, D. C. Licciardello, and T. V. Ramakrishnan, Phys. Rev. Lett. 42, 673 (1979).

${ }^{4}$ P. A. Lee and T. V. Ramakrishnan, Rev. Mod. Phys. 57, 287 (1985).

${ }^{5}$ G. Bergmann, Phys. Rep. 107, 1 (1984).

${ }^{6}$ B. L. Altshuler and A. G. Aronov, in Electron-Electron Interactions in Disordered Systems, edited by A. L. Efros and M.
Pollak (North-Holland, Amsterdam, 1985).

${ }^{7}$ A. Houghton, J. R. Senna, and S. C. Ying, Phys. Rev. B 25, 2196 (1982).

${ }^{8}$ L. D. Landau and E. M. Lifshitz, Quantum Mechanics (Pergamon, London, 1959), Ch. XVI.

${ }^{9}$ A. A. Abrikosov, L. P. Gor'kov, and I. E. Dzyaloshinsky, Methods of Quantum Field Theory in Statistical Physics (Prentice Hall, Englewood Cliffs, NJ, 1963), Chap. 7.

${ }^{10}$ Table of Integrals, Series and Products, edited by I. S. Gradshteyn and I. M. Ryzhik (Academic, New York, 1965). 